Amsterdam Expeditions to the West Indian Islands, Report 26*)

\title{
PREMIÈRE DÉCOUVERTE D'AMPHIPODES GAMMARIDAE DU GROUPEMENT DES HADZIIDES DANS DES EAUX SOUTERRAINES DE L'AMÉRIQUE DU SUD: DESCRIPTION DE METANIPHARGUS VENEZOLANUS SP. N.
}

\author{
par \\ JAN H. STOCK \& LAZARE BOTOSANEANU \\ Institut de Zoologie taxonomique, Université d'Amsterdam, Boîte postale 20125, \\ 1000 HC Amsterdam, Pays-Bas
}

\begin{abstract}
RÉSUMÉ
Description d'une nouvelle espèce de Metaniphargus, $M$. venezolanus, d'une grotte du Vénézuéla septentrional. C'est le premier représentant découvert en Amérique du Sud du groupement des Hadziides de la famille des Gammaridae. Il est étroitement apparenté à certains taxa insulaires des Caraibes.
\end{abstract}

\section{SUMMARY}

Description of a new species of Metaniphargus, $M$. venezolanus, from a cave in northern Venezuela. This is the first representative of the hadziid group of genera of the family Gammaridae to be found in South America. It is closely related to certain Caribbean, insular taxa.

\section{INTRODUCTION}

Les Gammaridae du groupement des Hadziides ("family group Hadziidae" selon l'opinion de Bousfield, 1977) sont connus d'une région qui correspond plus ou moins exactement à l'emplacement de la Mer Téthys pendant l'Oligocène (voir Stock, 1977, fig. 1). Les espèces, appartenant à 17 genres, sont distribuées dans la région périméditerranéenne, les îles Canaries, les Antilles, l'Amérique centrale (Mexique), le Texas, la Californie, et certaines îles du Pacifique ou de l'Océan Indien (îles Carolines, Hawaii, la Réunion). Les espèces ont été ren-

*) Le “Report no. 25" est publié dans la même livraison de ce journal. contrées pour la plupart dans les eaux souterraines "continentales", mais il y a également un nombre de formes connues de l'interstitiel marin.

Or, dans le cadre des Expéditions Amsterdamoises aux Indes Occidentales, plusieurs chercheurs de notre groupe de recherche ont prospecté la zone côtière du Vénézuéla, dans l'espoir d'y découvrir des taxa-sœurs des formes stygobies que l'on connaît des Antilles. Pendant ces prospections, on a découvert, dans l'eau d'une des grottes de la région karstique de Mayorquines (péninsule de Morocoy), un Metaniphargus nouveau, donc le premier représentant du groupement des Hadziides dans l'Amérique du Sud. Le genre Metaniphargus était jusqu'ici uniquement connu des îles des Caraïbes, où 11 espèces et sous-espèces distinctes sont reconnues à ce jour.

Les grottes de Mayorquines, d'où provient le premier Metaniphargus continental, sont localisées tout près de la mer des Caraïbes, mais contiennent des eaux pratiquement douces. Outre l'Amphipode que nous allons décrire, ces grottes ont donné une Cyathura (Isopoda, Anthuridae) stygobie, donc un représentant d'un autre groupe présent dans les Antilles et en Amérique centrale, mais pas encore signalé d'Amérique du Sud. Certes, les Crustacés stygobies de ces grottes ont une allure caraïbique plutôt que continentale. 


\section{Metaniphargus venezolanus sp. $n$.}

Matériel et localité. - Holotype (1 $\left.\sigma^{\circ}\right)$, allotype (1\&) et paratypes (22 $\left.\$, 50^{\circ} 0^{\circ}\right)$. Expéditions Amsterdamoises aux Indes Occidentales, sta. 82-512: Vénézuéla, Estado Falcón, Distrito Silva, Peninsula de Morocoy sur la côte Nord du pays, une des Cuevas de Mayorquines (coordonnées approximatives $10^{\circ} 53^{\prime} 27^{\prime \prime} \mathrm{N} 68^{\circ} 14^{\prime} 49^{\prime \prime} \mathrm{O}$ ). La localité la plus importante, près de la côte, pour aborder la péninsule est Chichiriviche. Le 1 et le 2 mars 1982; coll. L. Botosaneanu, N. Broodbakker, J. Notenboom et S. van Lieshout. (Musée Zoologique d'Amsterdam, coll. no. ZMA Amph. 107.577 a-c.)

Cette petite péninsule est en bonne partie calcaire, en contraste avec les zones avoisinantes, et juste à sa pointe ("Mayorquines") se développe un assez important réseau de grottes, verticales ou horizontales, dont quelques-unes donnent accès à un niveau phréatique. Un certain nombre de ces grottes ont été inventoriées dans Anonymus (1973a: 56-60) et dans Anonymus (1973b: 82-89). Lors de notre expédition de 1982, plusieurs grottes ont pu être explorées à Mayorquines, mais à cause du terrain difficile (le karst de Mayorquines est un véritable chaos!) il a rarement été possible d'assimiler ces grottes à celles inventoriées dans les publications mentionnées. Ainsi, la grotte ayant fourni l'espèce nouvelle de Metaniphargus est fort vraisemblablement une de celles que les spéléologues vénézuéliens n'ont pas encore inventoriées; ce qui est certain, c'est qu'elle se trouve à petite distance (ca. $60 \mathrm{~m}$ à vol d'oiseau en direction S.S.E.) de l'entrée d'une grotte (Cueva de los Petroglifos) bien connue par ses bas-reliefs indiens. L'entrée est à quelques mètres au-dessus du niveau de la mer.

Dans la grotte ayant fourni les Amphipodes, un niveau phréatique à l'obscurité totale est abordable après une assez raide descente; l'eau forme plusieurs "pools" assez peu profonds, entre de gros blocs clastiques, mais elle inonde aussi une diaclase longue de quelques dizaines de mètres, où elle peut atteindre plusieurs mètres de profondeur. L'eau est pratiquement douce en dépit de la proximité de la mer (chlorinité: $1024 \mathrm{mg} / \mathrm{l}$ ), absolument limpide, les ressources trophiques sont apparemment fort modestes (pas de matériel d'origine végétale, fort peu de guano de chauve-souris). La population de Metaniphargus doit être assez considérable.

Description. - Longueur maximum du corps (sans antennes et uropodes) $5,3 \mathrm{~mm}$. Dépigmenté, aveugle. Les appendices ne montrent pas de dimorphisme sexuel secondaire, à l'exception des gnathopodes 2 et des pléopodes 3.

Antenne supérieure (fig. 1) très allongée, aussi longue que le corps. Pédoncule de trois articles, dont le premier est $1 \frac{1 / 4}{4}$ fois plus long que le deuxième; le troisième article a la moitié de la longueur du deuxième article. Flagellum principal de 32 ou 33 articles chez les individus les plus grands, portant 8 ou 9 aesthètes, un sur chaque article distal sauf sur le dernier et sur l'avant-dernier article. Ces aesthètes (fig. 3) ont un peu plus de la moitié de la longueur de l'article correspondant du flagellum. Flagellum accessoire de 2 articles (fig. 2), presqu'aussi long que les deux articles proximaux du flagellum principal.

Antenne inférieure (fig. 4) beaucoup plus courte que l'antenne supérieure (40\% environ de sa longueur). Les articles 4 et 5 de son pédoncule sont minces et sveltes; le flagellum comprend 16 ou 17 articles chez les adultes.

Lèvre supérieure comme chez $M$. curasavicus Stephensen, 1933 (voir Stock, 1977, fig. 7e).

Mandibule droite (fig. 5) avec une soie longue sur la pars molaris, soie qui fait défaut sur la mandibule gauche (fig. 6). Lacinia mobilis droite bifide, avec 5 ou 6 dents fines; celle gauche simple, avec 5 dents plus robustes. Entre la lacinia et la pars molaris, on trouve 4 soies (dont 2 modifiées) sur l'appendice droit, 5 soies (dont 1 modifiée) sur celui gauche. Palpe de 3 articles, article 1 inerme, article 2 avec 1 soie terminale, article 3 avec une rangée de 10 soies " $D$ " environ, sensiblement de même taille, et avec 4 soies " $E$ ".

Lèvre inférieure (fig. 11) avec des lobes internes fort réduits.

Maxille antérieure (fig. 8) avec palpe asymétrique: celui de droite possède un article basal quasi-carré, et porte 5 éléments épais, spiniformes, et 1 soie fine sur l'article distal; celui de gauche (fig. 7) possède un article basal allongé, tandis que l'article distal en est armé de 4 éléments minces, chétiformes, et de 2 soies fines. Le lobe externe de la maxille antérieure est pourvu de 9 épines distales, dont la plus externe porte 9 ou 10 fins denticules médians; le nombre des denticules augmente graduellement dans le sens externe-interne, l'épine la plus interne ayant 16 de ces denticules environ (fig. $9)$. Le lobe interne porte 17 soies plumeuses.

Maxille postérieure (fig. 10): lobe externe avec 20 soies distales recourbées (11 plus longues que les 9 autres); lobe interne avec une 


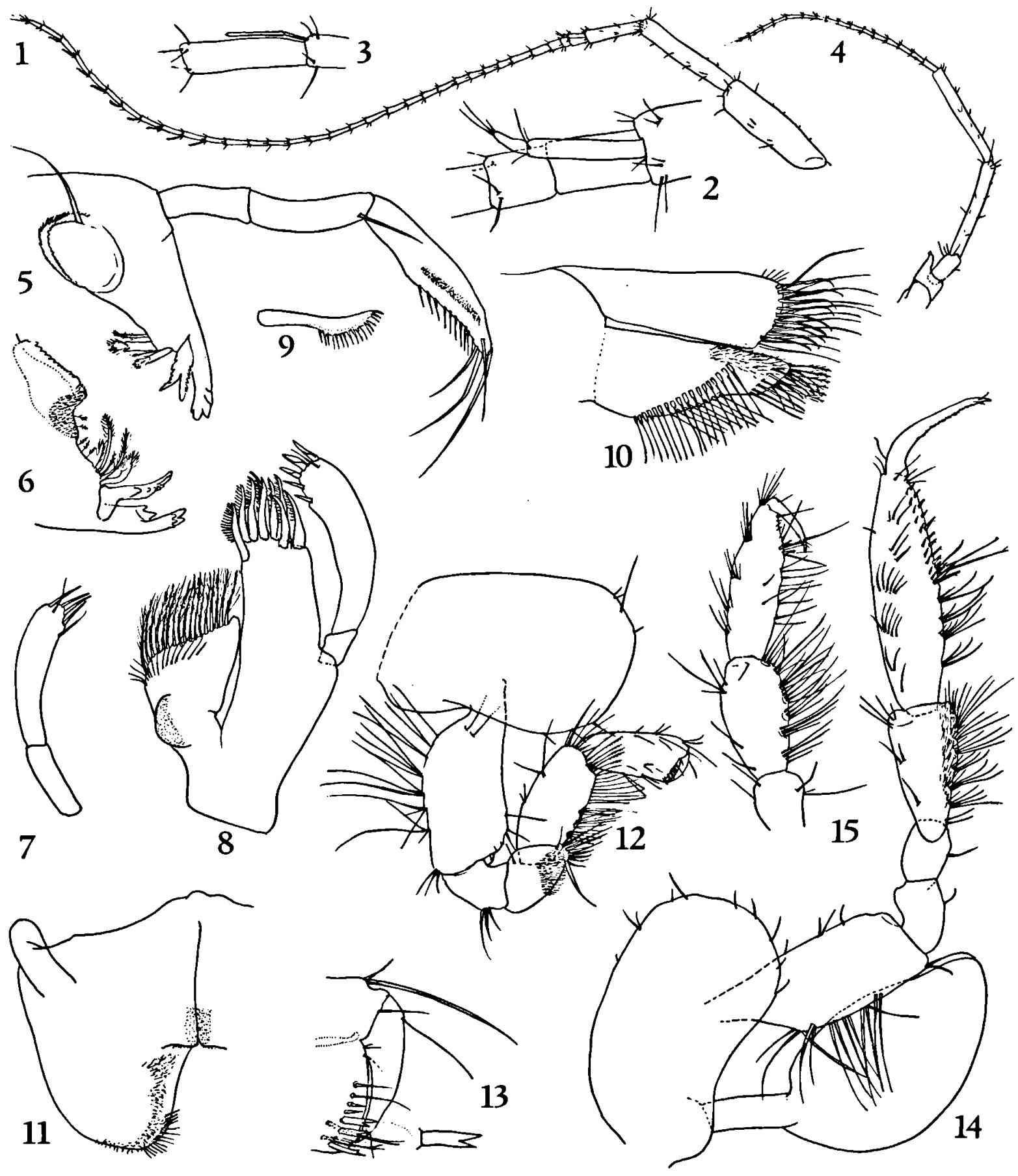

Figs. 1-15. Metaniphargus venezolanus sp. n. (paratypes): 1, antenne supérieure, $\sigma^{\prime \prime}$ (échelle A); 2 , flagellum accessoire, $\sigma^{\circ}$ (éch. B); 3, aesthète, $९$ (éch. B); 4, antenne inférieure, $\sigma^{*}$ (éch. A); 5, mandibule droite, $९$ (éch. B); 6, partie masticatoire de la mandibule gauche, $\$$ (éch. B); 7, palpe de la maxille antérieure gauche, $\$$ (éch. B); 8, maxille antérieure droite, $\$$ (éch. B); 9, deuxième épine (comptée à partir de l'intérieur) du lobe externe de la maxille antérieure, $\$$, plus fortement magnifiée; 10, maxille postérieure, $Q$ (éch. B); 11, lèvre inférieure, $Q$ (éch. B); 12, gnathopode 1, O (éch. C); 13, bord palmaire du gnathopode 1, $\sigma^{\circ}$ (éch. D); 14, gnathopode 2, O' (éch. C); 15, articles distaux du gnathopode 2, Q (éch. C). Les échelles figurent sur la planche suivante. 
rangée oblique de 18 soies, une rangée médiane de 8 soies, une rangée subdistale de 6 soies, et 2 rangées distales, chacune de 6 soies, dont 6 plumeuses et 6 lisses.

Maxillipède: ressemble beaucoup à celui de M. curasavicus (voir Stock, 1977, fig. 9a).

Gnathopode 1 (fig. 12) avec une plaque coxale assez allongée. Carpe très allongé, plus long que le propode, armé de 4 ou 5 groupes de soies longues sur son bord postérieur. Bord postérieur du propode avec 2 groupes de soies courtes; bord palmaire orné de 2 soies bifides et de 4 épines également bifides (fig. 13).

Gnathopode 2 sexuellement dimorphe. Articles proximaux identiques chez les deux sexes. Carpe du mâle légèrement plus triangulaire et moins allongé que celui de la femelle. Propode du mâle (fig. 14) plus grand et plus épais que celui de la femelle (fig. 15). Griffe terminale et bord palmaire plus longs chez le mâle que chez la femelle. Bord palmaire $O^{\circ}$ avec 2 épines fort longues sur l'angle et 2 rangées de petites épines, chacune de 11 épines environ. Bord palmaire $Q$ avec 2 épines sur l'angle et avec 2 soies longues et 2 ou 3 soies courtes.

Péréiopode 3 (fig. 16) avec plaque coxale assez allongée. Péréiopode 4 identique à $P 3$, plaque coxale (fig. 21) non émarginée.

Péréiopodes 5,6 et 7 allongés, armés presque uniquement d'épines. P5 (fig. 18) avec un article basal assez large, pas lobé; son bord postérieur porte 7 spinules environ; propode et griffe terminale très allongés et minces. P6 (fig. 19) et P7 (fig. 20) avec un article basal assez large, dont l'angle postéroventral est légèrement concave, inerme, pas lobé; le bord postérieur de cet article est serrulé, armé de 7 ou 8 spinules. Griffe de P7 moins allongée que celle de P6.

Branchies coxales (fig. 14) grandes, ovoïdes, avec un pédoncule long et très nettement délimité; elles sont associées aux pattes 2 à 6 . Oostégites (fig. 17) linéaires, portant généralement 6 ou 7 soies; associés aux pattes 2 à 5 .

Plaques épimérales (figs. 26, 27) au bord ventral droit; l'angle postéroventral acuminé en petite pointe.

Pléopodes normaux; pléopode 3 du mâle légèrement modifié (fig. 25): l'article basal de l'exopodite est rugueux et montre 2 faibles bosses sur son bord interne; cet article est apparemment homologue aux 2 articles proximaux de la femelle (fig. 24). L'article basal de l'endopodite est identique à celui de la femelle, avec un bord latéral régulièrement courbe.

Uropode 1 (fig. 28): pédoncule avec 1 épine ventrobasale; rame externe aux bords lisses, rame interne avec 1 épine marginale.

Uropode 2 (fig. 29): pédoncule distalement pourvu d'une rangée de 5 spinules.

Uropode 3 (fig. 30): rame externe biarticulée, article distal acuminé, sa longueur dépassant légèrement celle des épines terminales de l'article basal. La rame interne a $60 \%$ environ de la longueur de l'article basal de la rame externe. Les deux rames ne sont armées que d'épines.

Telson (figs. 22, 23) raccourci, composé de deux moitiés entièrement séparées; chaque moitié est arrondie dans sa partie basale, triangulaire dans sa partie distale. L'armature des lobes du telson est variable, non seulement entre des individus différents, mais aussi entre les moitiés droite et gauche d'un seul individu; le bord médian porte 0 ou 1 épine, le bord terminal-interne 1 ou 2 épines, le bord latéral 1 ou 2 épines. Dorsalement, les deux soies sensorielles habituelles s'insèrent sur les deux lobes du telson.

Affinités. - L'espèce nouvelle appartient, à l'intérieur du genre Metaniphargus, au groupe nicholsoni (voir Stock, 1977: 35), caractérisé par la combinaison suivante de caractères: (1) carpe du gnathopode $2(\%)$ très allongé; (2) bord palmaire du gnathopode 2 (\$) sétifêre; (3) pédoncule de l'uropode $2\left(\%, O^{*}\right)$ distalement pourvu d'une rangée de spinules; (4) pléopode $3\left(\sigma^{*}\right)$ sans modifications ou avec des modifications légères; (5) endopodite des uropodes $3\left(\%,{ }^{\circ}\right)$ dépourvu de soies plumeuses.

Cinq taxa appartiennent au groupe nicholsoni: M. nicholsoni Shoemaker, 1959 (de l'île Barbuda), M. palpator Stock, 1977 (des îles St. Martin et Anguilla), M. bousfieldi Stock, 1977 (de Puerto Rico), M. longipes longipes Stock, 1977 


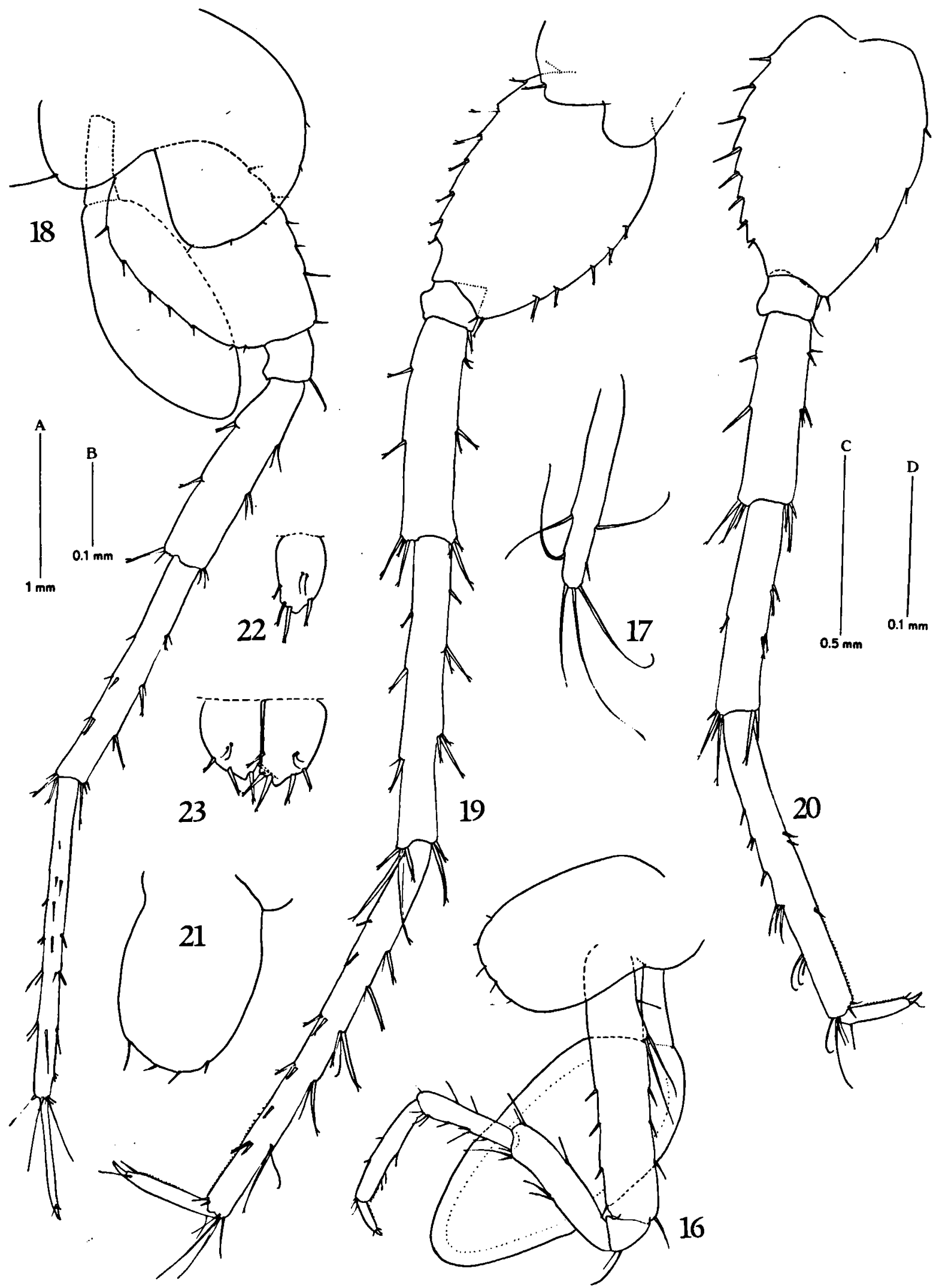

Figs. 16-23. Melaniphargus venezolanus sp. n. (paratypes): 16, péréiopode 3, 9 (échelle C); 17, oostégite du péréiopode 3 ,

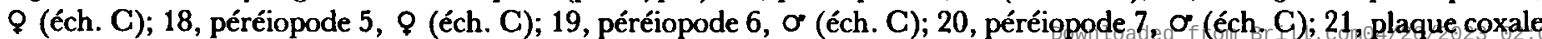
du péréiopode 4, 9 (éch. C); 22, moitié droite du telson, Ơ (éch. C); 23, telson d'un autre Ơ (éch. C). via free acces 


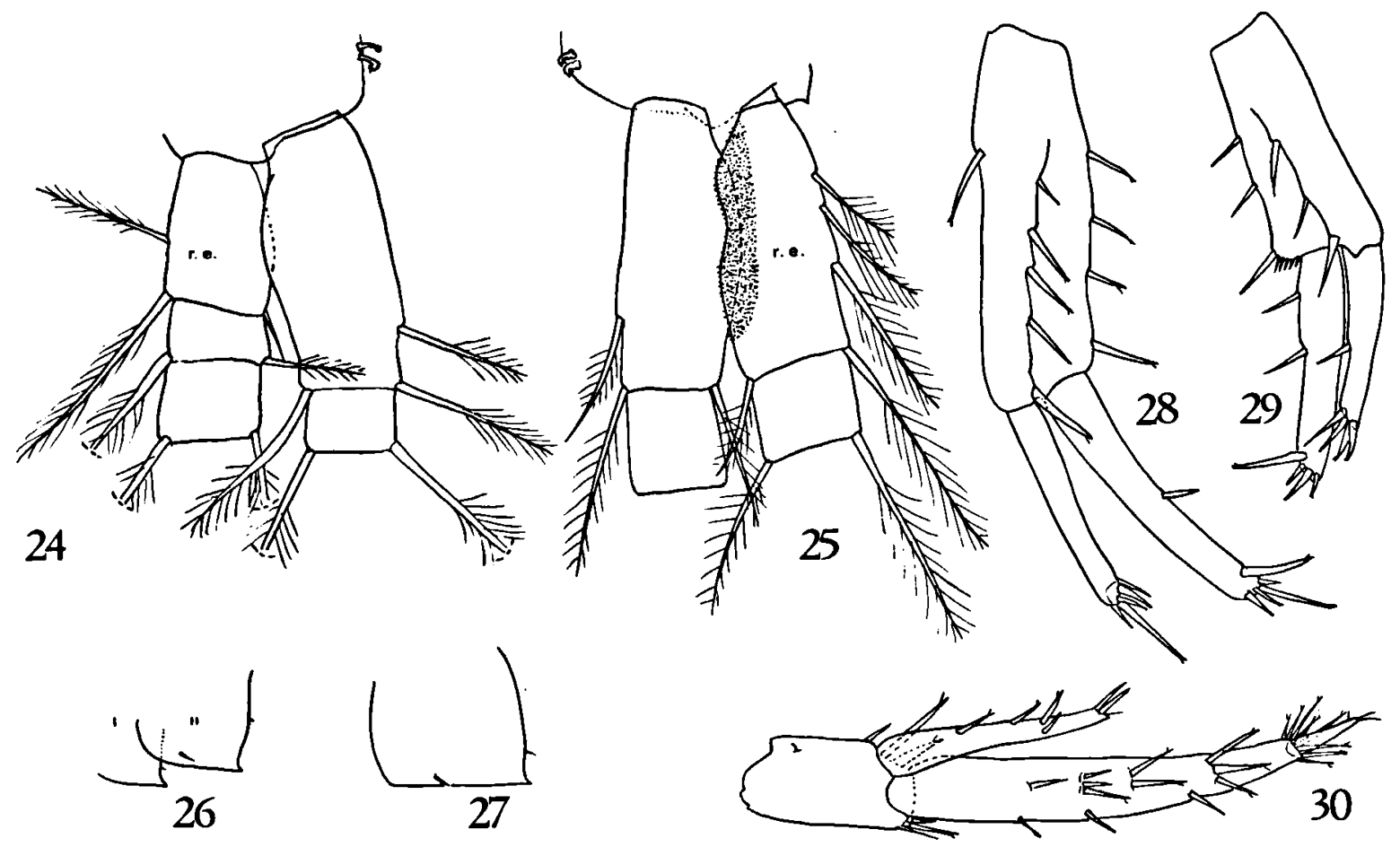

Figs. 24-30. Metaniphargus venezolanus sp. n. (paratypes): 24, partie basale du pléopode 3, 9 (échelle B); 25, partie basale du pléopode 3, O* (éch. B); 26, plaques épimérales I et II, O* (éch. A); 27, plaque épimérale III, O० (éch. A); 28, premier uropode, $\sigma^{\prime}$ (éch. C); 29, deuxième uropode, $\sigma^{*}$ (éch. C); 30, troisième uropode, $\sigma^{\circ}$ (éch. C). Les échelles figurent sur la planche précédente (r.e. = rame externe).

(d'Aruba) et $M$. longipes christophorensis Stock, 1977 (de la partie occidentale de Curaçao).

$M$. venezolanus peut être facilement distingué de toutes les espèces et sous-espèces du groupe nicholsoni par la forme du palpe de la maxille antérieure et par l'ornementation des épines terminales sur le lobe externe de celle-ci. Le palpe de $M x 1$ est plus allongé chez venezolanus que chez les autres taxa. Les épines du lobe externe sont munies d'un nombre plus grand de denticules chez venezolanus; par exemple, l'épine la plus latérale est munie de 9 à 10 denticules médians chez venezolanus, d'un nombre plus réduit (2, 3 ou 4 seulement) chez les autres taxa. Le nombre de denticules sur les épines terminales internes differe moins nettement: chez venezolanus il y en a 16 environ, chez les autres taxa 10 à 14.

Outre ces différences dans la maxille antérieure, on peut observer des points de distinction avec l'un ou l'autre des taxa:
- La nouvelle espèce se distingue facilement de $M$. nicholsoni par la forme de l'article basal des $\mathrm{P} 5$ à P7. Cet article possède un angle postéroventral lobé et proéminent chez l'espèce de Barbuda, non-lobé et même concave chez celle du Vénézuéla.

- Des différences avec $M$. bousfieldi se trouvent dans l'antenne supérieure (chez bousfieldi l'article 2 en est plus long que l'article 1), l'inégalité de taille entre les épines sur l'angle et celles sur le bord palmaire du gnathopode 2 du mâle (moins prononcée chez bousfieldi) et la présence d'une épine médiodorsale sur l'exopodite des premiers uropodes (absente chez venezolanus).

- M. palpator, connu de deux grottes sur St. Martin et Anguilla (faisant partie des Iles Surle-Vent, donc situées à une distance considérable de la côte du Vénézuéla), se distingue par son palpe mandibulaire (dont l'article distal montre un bord ventral tout droit), par la forme générale et l'angle postéroventral (plus pro- 
noncé) du basis des P6 et P7, par la griffe (plus longue) du gnathopode 2 du mâle et par la présence d'une épine médiodorsale sur l'exopodite des uropodes 1.

- L'espèce la plus proche de $M$. venezolanus est sans doute $M$. longipes, connue (en deux sousespèces) de nombreuses localités des Iles Sousle-Vent d'Aruba et Curaçao, localités qui sont (tout au moins en ligne directe) pas très éloignées de la zone de Mayorquines sur le continent. Les différences dans les gnathopodes 2 et dans les péréiopodes 6 et 7 , mentionnées cidessus pour $M$. palpator, sont présentes aussi entre $M$. longipes et $M$. venezolanus. En outre, l'espèce insulaire possède un flagellum d'A2 à 11 articles, contre 16 ou 17 articles chez l'espèce continentale; enfin, l'article distal de l'exopodite de l'uropode 3 est plus long (en rapport avec la longueur des épines terminales sur l'article proximal) chez longipes.

\section{REMERCIEMENTS}

Nos recherches au Vénézuéla ont été subventionnées par l'Amsterdamse Universiteitsvereniging, la Treub Maat- schappij (Utrecht), la Fondation Beijerinck-Popping (Amsterdam) et l'Université d'Amsterdam.

L'Ambassade des Pays-Bas à Caracas, et surtout son Attaché agricole, Dr. Ir.Th. P. M. de Wit, ont hautement contribué à la logistique de notre expédition.

Les auteurs tiennent à remercier la Sociedad Venezolana de Espeleologia (Caracas), et en particulier les Docteurs Franco Urbani et Omar Linares, pour leurs renseignements très utiles sur les grottes de Mayorquines.

Nous remercions les biologistes qui ont participé au travail sur le terrain (et qui sont mentionnés dans la section "Matériel et localité») pour leur activité appréciée.

\section{RÉFÉRENCES BIBLIOGRAPHIQUES}

Anonymus, 1973a. Catastro espeleologico de Venezuela. Boln. Soc. venez. Espeleol., 4 (1): 39-88.

- , 1973b. Catastro espeleologico de Venezuela, 1: 1-151 (Sociedad Venezolana de Espeleologia, Caracas).

Bousfield, E. L., 1977. A new look at the systematics of gammaroidean amphipods of the world. Crustaceana, Suppl. 4: 282-316.

STock, J. H., 1977. The taxonomy and zoogeography of hadziid Amphipoda, with emphasis on the West Indian taxa. Stud. Fauna Curaçao, 55: 1-130. 